PROCEEDINGS OF THE AMERICAN MATHEMATICAL SOCIETY

Volume 124, Number 10, October 1996

\title{
HEIGHTS OF ALGEBRAIC POINTS LYING ON CURVES OR HYPERSURFACES
}

\author{
WOLFGANG M. SCHMIDT
}

(Communicated by William W. Adams)

\begin{abstract}
Our first aim will be to give an explicit version of a generalization of the results of Zhang and Zagier on algebraic points $(x, y)$ with $x+y+1=0$. Secondly, we will show that distinct algebraic points lying on a given curve of certain type can be distinguished in terms of some height functions. Thirdly, we will derive a bound for the number of points on such a curve whose heights are under a given bound and whose coordinates lie in a multiplicative group of given rank.
\end{abstract}

\section{INTRODUCTION}

When $K$ is a number field, let $V=V(K)$ be the set of its places, and for $v \in V$ let $|\cdot|_{v}$ be the absolute value belonging to $v$ which extends the ordinary or a $p$-adic absolute value of $\mathbb{Q}$. Further set $\|x\|_{v}=|x|_{v}^{d_{v} / d}$ where $d$ is the degree of $K$ and $d_{v}$ is the local degree associated with $v$. The additive version of the product formula is that

$$
\sum_{v \in V} \log \|x\|_{v}=0
$$

for $x \in K^{\times}$. For such $x$ set

$$
h(x)=\sum_{v \in V} \max \left(0, \log \|x\|_{v}\right)=\frac{1}{2} \sum_{v \in V}\left|\log \|x\|_{v}\right|,
$$

where the second equality follows from the product formula. Then $h(x)$ is the absolute logarithmic height of $x$; it is independent of the number field $K$ in which $x$ is embedded.

It is an old conjecture of Lehmer [2] that when $x$ is of degree $d$ over $\mathbb{Q}$, and is not 0 or a root of 1 , then

$$
h(x)>c_{1} / d
$$

with an absolute constant $c_{1}>0$. The best result in this direction is due to Dobrowolski [1] and says that $h(x)>\left(c_{2} / d\right)(\log \log d / \log d)^{3}$ if $d \geqq 3$. The example $x=2^{1 / d}$ shows that $(0.1)$ would be best possible. In contrast, there is the following

Received by the editors March 27, 1995.

1991 Mathematics Subject Classification. Primary 11G30.

The author was supported in part by NSF grant DMS-9401426.

(C)1996 American Mathematical Society 
result of Zhang [8]: Suppose $x, y$ are algebraic but not 0 or cube roots of 1 , and satisfy

$$
x+y+1=0 .
$$

Then

$$
h(x)+h(y) \geqq c_{3}>0
$$

with an absolute constant $c_{3}$. Zagier [7] gave a more natural proof and determined the best value of the constant: $c_{3}=\frac{1}{2} \log ((1+\sqrt{5}) / 2)$. We will prove the following

Theorem 1. Let $F\left(X_{1}, \ldots, X_{n}\right)$ be a polynomial with rational coefficients. Let $x_{1}, \ldots, x_{n}$ be nonzero algebraic numbers with

$$
F\left(x_{1}, \ldots, x_{n}\right)=0, \quad F\left(1 / x_{1}, \ldots, 1 / x_{n}\right) \neq 0 .
$$

Then

$$
\sum_{i=1}^{n} h\left(x_{i}\right) \geqq c_{3}(F)>0 .
$$

When $F$ has total degree $f$ and coefficients in $\mathbb{Z}$ of maximum modulus $H$, we may take

$$
c_{3}(F)=1 /\left(2^{4 f+2 n} H\right) .
$$

S. Ahlgren pointed out to me that the theorem can be generalized to polynomials $F$ with coefficients in a number field with at least one real embedding.

When $n=2$ and $F(X, Y)=X+Y+1$, then $F(x, y)=F(1 / x, 1 / y)=0$ yields $x+y=-1, x y=1$, so that $x, y$ are the roots of $Z^{2}+Z+1$, hence are cube roots of 1 . Therefore Theorem 1 contains Zhang's Theorem.

Instead of the sum

$$
h_{s}(\underline{\underline{x}})=\sum_{i=1}^{n} h\left(x_{i}\right)
$$

we could have taken a more "sophisticated" function, such as, e.g.,

$$
h\left(x_{1}, \ldots, x_{n}\right)=\sum_{v \in V} \max \left(0, \log \left\|x_{1}\right\|_{v}, \ldots, \log \left\|x_{n}\right\|_{v}\right) .
$$

$h$ can be interpreted as the height of the point $\left(1: x_{1}: \cdots: x_{n}\right)$ in projective space $\mathbb{P}^{n}$, and one can formulate a result on heights of such points satisfying homogeneous polynomial equations. But the proofs are more conveniently done in the affine setting, and $h$ has the disadvantage (crucial in (ii) below) that it is not invariant under replacing $x_{1}, \ldots, x_{n}$ by $1 / x_{1}, \ldots, 1 / x_{n}$.

When $A$ is a subgroup of $\overline{\mathbb{Q}}^{\times}$, where $\overline{\mathbb{Q}}$ is the algebraic closure of $\mathbb{Q}$, let $A^{n}$ be the product group $A \times \cdots \times A$. Suppose $\underline{\underline{x}}=\left(x_{1}, \ldots, x_{n}\right), \underline{\underline{x}}^{\prime}=\left(x_{1}^{\prime}, \ldots, x_{n}^{\prime}\right), \ldots$ lie in $\left(\overline{\mathbb{Q}}^{\times}\right)^{n}$. In this group $\underline{\underline{x}} / \underline{\underline{x}}^{\prime}=\left(x_{1} / x_{1}^{\prime}, \ldots, x_{n} / x_{n}^{\prime}\right)$. We now set

$$
\delta\left(\underline{\underline{x}}, \underline{\underline{x}}^{\prime}\right)=h_{s}\left(\underline{\underline{x}} / \underline{\underline{x}}^{\prime}\right)=\sum_{i=1}^{n} h\left(x_{i} / x_{i}^{\prime}\right) .
$$

Since $h(1 / x)=h(x)$ and $h(x y) \leqq h(x)+h(y)$, we have

(i) $\delta\left(\underline{\underline{x}}, \underline{\underline{x^{\prime}}}\right) \geqq 0$, with equality precisely when $\underline{\underline{x}} / \underline{\underline{x}}^{\prime} \in U^{n}$, where $U$ is the group of roots of 1 .

(ii) $\delta\left(\underline{\underline{x}}, \underline{\underline{x}}^{\prime}\right)=\delta\left(\underline{\underline{x^{\prime}}}, \underline{\underline{x}}\right)$. 
(iii) $\delta\left(\underline{\underline{x}}, \underline{\underline{x}}^{\prime \prime}\right) \leqq \delta\left(\underline{\underline{x}}, \underline{\underline{x}}^{\prime}\right)+\delta\left(\underline{\underline{x}}^{\prime}, \underline{\underline{x}}^{\prime \prime}\right)$.

Thus $\delta$ induces a metric on the factor group $\left(\bar{Q}^{\times}\right)^{n} / U^{n}$.

In what follows we will have $n=2$ and we will have $\underline{\underline{x}}=(x, y), \underline{\underline{x}}^{\prime}=\left(x^{\prime}, y^{\prime}\right), \ldots$ in $\left(\overline{\mathbb{Q}}^{\times}\right)^{2}$. Let $P \in \mathbb{C}[X, Y]$ be not divisible by $X$ or $Y$. Let $\mathcal{M}=\overline{\bar{M}}(P)$ be the set of monomials $X^{i} Y^{j}$ occurring in $P$ with nonzero coefficients, so that $P=\sum_{M \in \mathcal{M}} u_{M} M$ with coefficients $u_{M} \neq 0$. Suppose $P$ has respective degrees $a, b$ in $X, Y$, and set

$$
\widetilde{P}=\sum_{M \in \mathcal{M}} u_{M} X^{a} Y^{b} M^{-1}
$$

It is easily seen that $\widetilde{P}$ (like $P$ ) is not divisible by $X$ or $Y$ and is again of respective degrees $a, b$. Thus $\widetilde{\widetilde{P}}=P$, and the relation between $P$ and $\widetilde{P}$ is symmetric. Call $P$ reflexive if $\mathcal{M}(P)=\mathcal{M}(\widetilde{P})$. For example, $P=u X+v Y+w$ with $u v w \neq 0$ is not reflexive, but $P=s X Y+u X+v Y+w$ with suvw $\neq 0$ is reflexive.

Theorem 2. Suppose $P(X, Y)$ as above is irreducible and not reflexive. Let $P, \widetilde{P}$ be of respective total degrees $p, \tilde{p}$, and set $q=p \tilde{p}$. Suppose $\underline{\underline{x}}_{0}, \underline{\underline{x}}_{1}, \ldots, \underline{\underline{x}}_{q}$ are distinct zeros of $P$ lying in $\left(\overline{\mathbb{Q}}^{\times}\right)^{2}$. Then

$$
\sum_{i=1}^{q} \delta\left(\underline{\underline{x}}_{0}, \underline{\underline{x}}_{i}\right) \geqq c_{4}(P)>0 .
$$

When $\mathcal{M}(P)$ has cardinality $m$, we may take

$$
c_{4}(P)=2^{-8 p m}
$$

For a linear polynomial $P$, a related result had been proved by Schlickewei and Wirsing [5].

Finally we have

Theorem 3. Let $P(X, Y)$ be as in Theorem 2, and $\Gamma$ a subgroup of $\left(\bar{Q}^{\times}\right)^{2}$ containing at most $r$ multiplicatively independent elements. Then the number of zeros $\underline{\underline{x}} \in \Gamma$ of $P$ with $h(\underline{\underline{x}}) \leqq C$, where $C \geqq 1$, is

$$
\leqq c_{5}(P)^{r+1} C^{r}
$$

We may take

$$
c_{5}(P)=2^{9 p m}
$$

In the case of a linear polynomial $P$, a bound with $r^{2}$ in the exponent is implicit in Schlickewei [3].

Added in proof. Since submission of this paper, further work was done by E. Bombieri and U. Zannier in Algebraic Points on Subvarieties of $\mathbb{G}_{m}^{n}$, which has already appeared in International Mathematical Research Notices 7 (1995), 333-347. See also W. Schmidt, Heights of Points on Subvarieties of $\mathbb{G}_{m}^{n}$ (to appear in Proceedings Séminaire de Théorie des Nombres de Paris (1994/94)) and F. Beukers and D. Zagier, Lower bounds of heights of points on hypersurfaces (Preprint \#943, Univ. Utrecht, 1996). 


\section{Proof of Theorem 1}

Lemma 1. Let $x_{1}, \ldots, x_{n}$ be nonzero complex numbers, and set

$$
\rho=\max _{i}|1-| x_{i}||
$$

Then when $\mu>1$ we have

$$
\log \rho+\log \mu \leqq(e \log 2)^{-1} \mu \max _{i}|\log | x_{i}|| .
$$

Proof. Since we interpret $\log 0$ to be $-\infty$, we may suppose that $\rho \neq 0$. Set $\nu=\mu \rho$. We distinguish three cases.

(i) $0<\nu \leqq 1$. Here we observe that the left hand side of (1.1) is $\leqq 0$.

(ii) $1<\nu<\mu$. Pick $i$ with $|1-| x_{i}||=\rho=\nu / \mu$. Then $\left|x_{i}\right|=1 \pm \nu / \mu$, so that $|\log | x_{i}|| \geqq(\nu / \mu) \log 2$. Now

$$
\log \rho+\log \mu=\log \nu \leqq \nu / e \leqq(e \log 2)^{-1} \mu|\log | x_{i}||,
$$

and (1.1) holds.

(iii) $\nu \geqq \mu$. Then $\rho \geqq 1$, so that $\rho=\left|x_{i}\right|-1$ for some $i$, and the right hand side of (1.1) is $\geqq(e \log 2)^{-1} \mu \log (\rho+1)$. The desired assertion now follows from

$$
(e \log 2)^{-1} \mu \log (\rho+1)-\log \rho-\log \mu \geqq(e \log 2)^{-1} \mu \log 2-\log \mu \geqq 0 .
$$

Now let $\underline{\underline{x}}=\left(x_{1}, \ldots, x_{n}\right)$ be as in Theorem 1 , and set $K=\mathbb{Q}\left(x_{1}, \ldots, x_{n}\right)$ and $V(K)=V \stackrel{\stackrel{ }{=}}{=} V_{\infty} \cup V_{0}$, where $V_{\infty}, V_{0}$ consist of Archimedean and non-Archimedean places, respectively. For $v \in V$ set

$$
B_{v}=\max \left(0, \log \left|x_{1}\right|_{v}, \ldots, \log \left|x_{n}\right|_{v}\right),
$$

so that

$$
\max \left(1,\left|x_{1}\right|_{v}, \ldots,\left|x_{n}\right|_{v}\right)=e^{B_{v}}
$$

and

$$
\begin{aligned}
\sum_{v \in V} d_{v} B_{v} & =\sum_{v \in V} \max \left(0, d \log \left\|x_{1}\right\|_{v}, \ldots, d \log \left\|x_{n}\right\|_{v}\right) \\
& \leqq d \sum_{i=1}^{n} h\left(x_{i}\right)=d h_{s}(\underline{\underline{x}}) .
\end{aligned}
$$

Set

$$
\rho_{v}=\left.\max _{i}|1-| x_{i}\right|_{v} \mid \quad(v \in V)
$$

Write

$$
\widetilde{F}\left(X_{1}, \ldots, X_{n}\right)=X_{1}^{r_{1}} \cdots X_{n}^{r_{n}} F\left(1 / X_{1}, \ldots, 1 / X_{n}\right)
$$

where $r_{1}, \ldots, r_{n}$ are the respective degrees of $F$ in $X_{1}, \ldots, X_{n}$. Then $\widetilde{F}$ is a polynomial of total degree $\tilde{f} \leqq n f$. Our hypothesis implies that

$$
\widetilde{F}(\underline{\underline{x}}) \neq 0
$$

\section{Lemma 2.}

$$
\log |\widetilde{F}(\underline{\underline{x}})|_{v} \leqq\left\{\begin{array}{l}
n f B_{v} \text { when } v \in V_{0}, \\
(n+1) f B_{v}+\log \left(2^{4 f+2 n-2} H\right)+\log \rho_{v} \text { when } v \in V_{\infty} .
\end{array}\right.
$$


Proof. When $v \in V_{0}$, then

$$
|\widetilde{F}(\underline{\underline{x}})|_{v} \leqq e^{\tilde{f} B_{v}} \leqq e^{n f B_{v}},
$$

whence the assertion.

Suppose $v \in V_{\infty}$. We may assume that $K$ is embedded in $\mathbb{C}$, and $|\cdot|_{v}$ is the ordinary absolute value. We denote the complex conjugate of a number $z$ by $\bar{z}$. By the Taylor expansion about $\underline{\underline{x}}$ we have

$$
F\left(1 / \bar{x}_{1}, \ldots, 1 / \bar{x}_{n}\right)=\sum_{\underline{\underline{k}}} c_{\underline{\underline{k}}}\left(\left(1 / \bar{x}_{1}\right)-x_{1}\right)^{k_{1}} \cdots\left(\left(1 / \bar{x}_{n}\right)-x_{n}\right)^{k_{n}}
$$

where in view of $F(\underline{\underline{x}})=0$ the sum is over $n$-tuples $\underline{\underline{k}}=\left(k_{1}, \ldots, k_{n}\right) \neq \underline{\underline{0}}$ with $k_{i} \geqq 0$ $(i=1, \ldots, n), k_{1}+\cdots+k_{n} \leqq f$, and where $c_{\underline{\underline{k}}}=\overline{\overline{F_{\underline{k}}}}(\underline{\underline{x}})$ with

$$
F_{\underline{\underline{k}}}=\frac{1}{k_{1} ! \cdots k_{n} !} \frac{\partial^{k_{1}+\cdots+k_{n}}}{\partial X_{1}^{k_{1}} \cdots \partial X_{n}^{k_{n}}} F \text {. }
$$

The coefficients of $F_{\underline{\underline{k}}}$ have modulus $\leqq 2^{f} H$ (see, e.g., [6, Ch. V, Lemma 5A]). The number of monomials in $F_{\underline{\underline{k}}}$ is $\leqq\left(\begin{array}{c}f+n \\ n\end{array}\right) \leqq 2^{f+n-1}$, so that by (1.2),

$$
\left|c_{\underline{\underline{k}}}\right| \leqq 2^{2 f+n-1} H e^{B\left(f-k_{1}-\cdots-k_{n}\right)}
$$

with $B=B_{v}$. After multiplication by $\bar{x}_{1}^{r_{1}} \cdots \bar{x}_{n}^{r_{n}}$ we obtain

$$
\widetilde{F}(\underline{\underline{\underline{x}}})=\sum_{\underline{\underline{k}}} c_{\underline{\underline{k}}} \bar{x}_{1}^{r_{1}-k_{1}}\left(1-\left|x_{1}\right|^{2}\right)^{k_{1}} \cdots \bar{x}_{n}^{r_{n}-k_{n}}\left(1-\left|x_{n}\right|^{2}\right)^{k_{n}} .
$$

For $1 \leqq i \leqq n$,

$$
\left|\bar{x}_{i}\right|^{r_{i}-k_{i}}\left(1-\left|x_{i}\right|^{2}\right)^{k_{i}} \leqq 2^{k_{i}} \max \left(1,\left|x_{i}\right|\right)^{r_{i}+k_{i}} \leqq 2^{k_{i}} e^{\left(r_{i}+k_{i}\right) B} .
$$

But when $k_{i}>0$ we have with $\rho=\rho_{v}$ that

$$
\left|\bar{x}_{i}\right|^{r_{i}-k_{i}}\left(1-\left|x_{i}\right|^{2}\right)^{k_{i}} \leqq 2^{k_{i}} \rho \max \left(1,\left|x_{i}\right|\right)^{r_{i}+k_{i}-1} \leqq 2^{k_{i}} \rho e^{\left(r_{i}+k_{i}\right) B} .
$$

Thus (since the sum in (1.6) is over $\underline{\underline{k}} \neq \underline{\underline{0}}$ ),

$$
\begin{aligned}
|\widetilde{F}(\underline{\underline{x}})|=|\widetilde{F}(\underline{\underline{x}})| & \leqq \rho \cdot 2^{f} \sum_{\underline{\underline{k}}}\left|c_{\underline{\underline{k}}}\right| e^{\left(n f+k_{1}+\cdots+k_{n}\right) B} \\
& \leqq \rho \cdot 2^{f}\left(\begin{array}{c}
f+n \\
n
\end{array}\right) \cdot 2^{2 f+n-1} H e^{(n+1) f B} \\
& \leqq \rho \cdot 2^{4 f+2 n-2} H e^{(n+1) f B} .
\end{aligned}
$$

Therefore

$$
\log |\widetilde{F}(\underline{\underline{x}})| \leqq \log \rho+\log \left(2^{4 f+2 n-2} H\right)+(n+1) f B,
$$

and Lemma 2 is established.

We now multiply the inequality of the lemma by $d_{v}$ and take the sum over $v \in V$. The left hand side will become zero by (1.5) and the product formula. In view of (1.3) we obtain

$$
0 \leqq(n+1) f d h_{s}(\underline{\underline{x}})+d \log \left(2^{4 f+2 n-2} H\right)+\sum_{v \in V_{\infty}} d_{v} \log \rho_{v} .
$$


When $\mu>10$, Lemma 1 yields

$$
\begin{aligned}
& \sum_{v \in V_{\infty}} d_{v} \log \rho_{v}=-d \log \mu+\sum_{v \in V_{\infty}} d_{v}\left(\log \rho_{v}+\log \mu\right) \\
& \leqq-d \log \mu+\left.(e \log 2)^{-1} \mu \sum_{v \in V_{\infty}} d_{v} \max _{i}|\log | x_{i}\right|_{v} \mid \\
& =-d \log \mu+(e \log 2)^{-1} \mu d \sum_{v \in V_{\infty}} \max _{i}\left|\log \left\|x_{i}\right\|_{v}\right| .
\end{aligned}
$$

But

$$
\sum_{v \in V_{\infty}} \max _{i}\left|\log \left\|x_{i}\right\|_{v}\right| \leqq \sum_{i=1}^{n} \sum_{v \in V}\left|\log \left\|x_{i}\right\|_{v}\right|=2 h_{s}(\underline{\underline{x}}) .
$$

If we substitute all this into (1.7) and divide by $d$, we get

$$
0 \leqq-\log \mu+((n+1) f+(2 / e \log 2) \mu) h_{s}(\underline{\underline{x}})+\log \left(2^{4 f+2 n-2} H\right) .
$$

We set

$$
\mu=e \cdot 2^{4 f+2 n-2} H
$$

Since $f \geqq 1$, the coefficient of $h_{s}(\underline{\underline{x}})$ in $(1.10)$ is

$$
\begin{aligned}
(n+1) f+(2 / e \log 2) \mu & \leqq\left((n+1) \cdot 2^{-4} \cdot 2^{4 f}+(1 / \log 2) \cdot 2^{4 f+2 n-1}\right) H \\
& <2^{4 f+2 n} H .
\end{aligned}
$$

Thus indeed $h_{s}(\underline{\underline{x}})>1 /\left(2^{4 f+2 n} H\right)$.

For later applications we will prove the following

Theorem 1a. When $n=4$ and

$$
F_{0}\left(X_{1}, X_{2}, X_{3}, X_{4}\right)=\left|\begin{array}{ccc}
1 & 1 & 1 \\
1 & X_{1} & X_{2} \\
1 & X_{3} & X_{4}
\end{array}\right|,
$$

we may take $c_{3}\left(F_{0}\right)=1 / 52$.

We begin with

Lemma 2a. In the situation of Theorem 1a,

$$
\log \left|\widetilde{F}_{0}(\underline{\underline{x}})\right|_{v} \leqq\left\{\begin{array}{l}
3 B_{v} \quad \text { when } \quad v \in V_{0}, \\
5 B_{v}+4 \log 2+\log \rho_{v}
\end{array} \text { when } v \in V_{\infty} .\right.
$$

Proof. Note that

$$
\begin{aligned}
\widetilde{F}_{0}\left(X_{1}, X_{2}, X_{3}, X_{4}\right)= & X_{2} X_{3}-X_{1} X_{4}+X_{1} X_{3} X_{4}+X_{1} X_{2} X_{4} \\
& -X_{2} X_{3} X_{4}-X_{1} X_{2} X_{3} .
\end{aligned}
$$

The assertion for $v \in V_{0}$ follows immediately. On the other hand, as a special case of (1.6),

$$
\begin{aligned}
\widetilde{F}_{0}(\underline{\underline{x}})= & \left(x_{4}-1\right)\left(1-\left|x_{1}\right|^{2}\right) \bar{x}_{2} \bar{x}_{3} \bar{x}_{4}+\left(x_{1}-1\right)\left(1-\left|x_{4}\right|^{2}\right) \bar{x}_{1} \bar{x}_{2} \bar{x}_{3} \\
& -\left(x_{3}-1\right)\left(1-\left|x_{2}\right|^{2}\right) \bar{x}_{1} \bar{x}_{3} \bar{x}_{4}-\left(x_{2}-1\right)\left(1-\left|x_{3}\right|^{2}\right) \bar{x}_{1} \bar{x}_{2} \bar{x}_{4} \\
& +\left(1-\left|x_{1}\right|^{2}\right)\left(1-\left|x_{4}\right|^{2}\right) \bar{x}_{2} \bar{x}_{3}-\left(1-\left|x_{2}\right|^{2}\right)\left(1-\left|x_{3}\right|^{2}\right) \bar{x}_{1} \bar{x}_{4} .
\end{aligned}
$$


The right hand side equals

$$
\begin{aligned}
& \left(1-\left|x_{1}\right|^{2}\right) \bar{x}_{2} \bar{x}_{3}\left(\frac{1}{4}\left|x_{4}\right|^{2}+\frac{1}{2}-\bar{x}_{4}\right)+\left(1-\left|x_{4}\right|^{2}\right) \bar{x}_{2} \bar{x}_{3}\left(\frac{1}{2}\left|x_{1}\right|^{2}+\frac{1}{2}-\bar{x}_{1}\right) \\
& -\left(1-\left|x_{2}\right|^{2}\right) \bar{x}_{1} \bar{x}_{4}\left(\frac{1}{2}\left|x_{3}\right|^{2}+\frac{1}{2}-\bar{x}_{3}\right)+\left(1-\left|x_{3}\right|^{2}\right) \bar{x}_{1} \bar{x}_{4}\left(\frac{1}{2}\left|x_{2}\right|^{2}+\frac{1}{2}-\bar{x}_{2}\right) .
\end{aligned}
$$

Now the sum of the moduli of the coefficients in $(1+X)\left(\frac{1}{2} Y^{2}+\frac{1}{2}-Y\right)$ is 4 . Therefore we obtain

$$
\left|\widetilde{F}_{0}(\underline{\underline{x}})\right|=\left|\widetilde{F}_{0}(\underline{\underline{x}})\right| \leqq 4 \rho \cdot 4 e^{5 B}=e^{5 B} \cdot 2^{4} \rho,
$$

and Lemma 2a follows.

If we take the inequalities of Lemma $2 \mathrm{a}$, multiply by $d_{v}$, and take the sum over $v \in V$, we obtain

$$
0 \leqq 5 d h_{s}(\underline{\underline{x}})+4 d \log 2+\sum_{v \in V_{\infty}} d_{v} \log \rho_{v}
$$

In view of (1.8), (1.9) we obtain after division by $d$ that

$$
0 \leqq-\log \mu+(5+(2 / e \log 2) \mu) h_{s}(\underline{\underline{x}})+4 \log 2 .
$$

We now take $\mu=e \cdot 2^{4}$, so that

$$
5+(2 / e \log 2) \mu=5+2^{5} / \log 2<52,
$$

and $(1.10 \mathrm{a})$ gives $h_{s}(\underline{\underline{x}})>1 / 52$.

\section{Proof of Theorem 2}

Write

$$
P=\sum_{k=1}^{m} u_{k} M_{k}
$$

with distinct monomials $M_{k}$ and nonzero coefficients $u_{k}$. Since $P$ is nonreflexive, $\mathcal{M}(P) \nsubseteq \mathcal{M}(\widetilde{P})$. By hypothesis, $P\left(\underline{\underline{x}}_{i}\right)=0$, i.e., $\sum_{k=1}^{m} u_{k} M_{k}\left(\underline{\underline{x}}_{i}\right)=0(i=0, \ldots, q)$, so that the matrix

$$
\left(M_{k}\left(\underline{\underline{x}}_{i}\right)\right) \quad(1 \leqq k \leqq m, 0 \leqq i \leqq q)
$$

has rank $<m$. If we divide the $k$-th row by $M_{k}\left(\underline{\underline{x}}_{0}\right)$ we obtain the matrix

$$
\left(M_{k}\left(\underline{\underline{x}}_{i} / \underline{\underline{x}}_{0}\right)\right) \quad(1 \leqq k \leqq m, 0 \leqq i \leqq q),
$$

which is also of rank $<m$.

Next, consider the matrix

$$
\left(M_{k}\left(\underline{\underline{x}}_{0} / \underline{\underline{x}}_{i}\right)\right) \quad(1 \leqq k \leqq m, 0 \leqq i \leqq q) .
$$

Suppose this matrix also had rank $<m$. Then there are relations

$$
\sum_{k=1}^{m} w_{k} M_{k}\left(\underline{\underline{x}}_{0} / \underline{\underline{x}}_{i}\right)=\sum_{k=1}^{m} w_{k} M_{k}\left(\underline{\underline{x}}_{0}\right) / M_{k}\left(\underline{\underline{x}}_{i}\right)=0 \quad(0 \leqq i \leqq q)
$$

where not all the coefficients $w_{k}$ are zero. Thus the polynomial

$$
\widehat{P}(\underline{\underline{X}})=\sum_{k=1}^{m} w_{k} M_{k}\left(\underline{\underline{x}}_{0}\right) X^{a} Y^{b} M_{k}(\underline{\underline{X}})^{-1}
$$


(where again $a, b$ are the degrees of $P$ in $X, Y$ ) vanishes at $\underline{\underline{x}}_{0}, \ldots, \underline{\underline{x}}_{q}$. Clearly $\mathcal{M}(\widehat{P}) \subseteq \mathcal{M}(\widetilde{P})$, so that $\mathcal{M}(P) \nsubseteq \mathcal{M}(\widehat{P})$, and $P, \widehat{P}$ are not constant multiples of each other. Since $\widehat{P}$ has respective degrees $\leqq a, b$ in $X, Y$, it is not a multiple of $P$. Since $P$ is irreducible, $P, \widehat{P}$ have no common factor. Their respective total degrees are $p$ and $\leqq \tilde{p}$, so that by Bezout's Theorem they have at most $q=p \tilde{p}$ common zeros, contradicting the fact that they have the zeros $\underline{\underline{x}}_{0}, \underline{\underline{x}}_{1}, \ldots, \underline{\underline{x}}_{q}$.

We may conclude that the matrix (2.2) has rank $m$. There are integers $i_{0}, i_{1}, \ldots$, $i_{m-1}$ such that the matrix

$$
\left(M_{k}\left(\underline{\underline{x}}_{0} / \underline{\underline{x}}_{i}\right)\right) \quad(1 \leqq k \leqq m, 0 \leqq t<m)
$$

is nonsingular. We clearly may pick $i_{0}, \ldots, i_{m-1}$ with $i_{0}=0$. Then the matrix with rows

$$
\left(1, M_{k}\left(\underline{\underline{x}}_{0} / \underline{\underline{x}}_{i_{1}}\right), \ldots, M_{k}\left(\underline{\underline{x}}_{0} / \underline{\underline{x}}_{i_{m-1}}\right)\right) \quad(1 \leqq k \leqq m)
$$

is nonsingular. Let $F\left(\underline{\underline{X}}_{1}, \ldots, \underline{\underline{X}}_{m-1}\right)$ with $\underline{\underline{X}}_{i}=\left(X_{i}, Y_{i}\right)$ be the determinant with rows

$$
\left(1, M_{k}\left(\underline{\underline{X}}_{1}\right), \ldots, M_{k}\left(\underline{\underline{X}}_{m-1}\right)\right) \quad(1 \leqq k \leqq m) .
$$

Then $F$ is a polynomial in $n=2(m-1)$ variables of total degree $f \leqq \operatorname{deg} M_{1}+$ $\cdots+\operatorname{deg} M_{m} \leqq m p$. By the nonsingularity of the matrix with rows (2.3),

$$
F\left(\underline{\underline{x}}_{0} / \underline{\underline{x}}_{i_{1}}, \ldots, \underline{\underline{x}}_{0} / \underline{\underline{x}}_{i_{m-1}}\right) \neq 0 .
$$

On the other hand, since (2.1) is of rank $<m$, the matrix with rows

$$
\left(1, M_{k}\left(\underline{\underline{x}}_{i} / \underline{\underline{x}}_{0}\right), \ldots, M_{k}\left(\underline{\underline{x}}_{i_{m-1}} / \underline{\underline{x}}_{0}\right)\right) \quad(1 \leqq k \leqq m)
$$

is singular, so that

$$
F\left(\underline{\underline{x}}_{i_{1}} / \underline{\underline{x}}_{0}, \ldots, \underline{\underline{x}}_{i_{m-1}} / \underline{\underline{x}}_{0}\right)=0 .
$$

By Theorem 1 , the point $\mathfrak{x}=\left(x_{i_{1}} / x_{0}, y_{i_{1}} / y_{0}, \ldots, x_{i_{m-1}} / x_{0}, y_{i_{m-1}} / y_{0}\right)$ has $h_{s}(\mathfrak{x}) \geqq$ $c_{3}(F)$. Then

$$
\sum_{i=1}^{q} \delta\left(\underline{\underline{x}}_{0}, \underline{\underline{x}}_{i}\right)=\sum_{i=1}^{q}\left(h\left(x_{i} / x_{0}\right)+h\left(y_{i} / y_{0}\right)\right) \geqq h_{s}(\mathfrak{x}) \geqq c_{3}(F) .
$$

Now $4 f+2 n \leqq 4 m p+2 \cdot 2(m-1) \leqq 8 p m-4$, so that we may take $c_{3}(F)=2^{-8 p m+4}$. Therefore Theorem 2 is true with $c_{4}(P)=2^{-8 p m+4}>2^{-8 p m}$.

Theorem 2a. When $P_{0}=u X+v Y+w$ with nonzero coefficients, we may take

$$
c_{4}\left(P_{0}\right)=1 / 52 .
$$

Proof. In this special case we have $m=3, M_{1}=1, M_{2}=X, M_{3}=Y$. Further $F\left(\underline{\underline{X}}_{1}, \underline{\underline{X}}_{2}\right)$ becomes

$$
\left|\begin{array}{ccc}
1 & 1 & 1 \\
1 & X_{1} & X_{2} \\
1 & Y_{1} & Y_{2}
\end{array}\right|,
$$

so that we may take $c_{4}(P)=c_{3}(F)=1 / 52$. 


\section{Proof of Theorem 3}

Suppose initially that $\Gamma$ is finitely generated and of rank $r$. There are $\underline{\underline{\alpha}}_{1}=$ $\left(\alpha_{1}, \beta_{1}\right), \ldots, \underline{\underline{\alpha}}_{r}=\left(\alpha_{r}, \beta_{r}\right)$, so that the elements of $\Gamma$ are

$$
\underline{\underline{x}}=(x, y)=\underline{\underline{\zeta}}_{1}^{u_{1}} \cdots \underline{\underline{\alpha}}_{r}^{u_{r}}=\left(\xi \alpha_{1}^{u_{1}} \cdots \alpha_{r}^{u_{r}}, \eta \beta_{1}^{u_{1}} \cdots \beta_{r}^{u_{r}}\right)
$$

where $\underline{\zeta}=(\xi, \eta) \in U^{2}$, and $\underline{\underline{u}}=\left(u_{1}, \ldots, u_{r}\right)$ runs through $\mathbb{Z}^{r}$. Here $\Gamma$, hence the $\alpha_{i}, \beta_{i}$ lie in a number field $K$. For $v \in V=V(K)$ put

$$
\begin{aligned}
a_{i v} & =\log \left\|\alpha_{i}\right\|_{v}, \quad b_{i v}=\log \left\|\beta_{i}\right\|_{v} \quad(i=1, \ldots, r), \\
a_{v}(\underline{\underline{\xi}}) & =\sum_{i=1}^{r} a_{i v} \xi_{i}, \quad b_{v}(\underline{\underline{\xi}})=\sum_{i=1}^{r} b_{i v} \xi_{i}
\end{aligned}
$$

where $\underline{\underline{\xi}}=\left(\xi_{1}, \ldots, \xi_{r}\right) \in \mathbb{R}^{r}$. Set

$$
\psi(\underline{\underline{\xi}})=\frac{1}{2} \sum_{v \in V}\left(\left|a_{v}(\underline{\underline{\xi}})\right|+\left|b_{v}(\underline{\underline{\xi}})\right|\right) .
$$

When $\underline{\underline{u}} \in \mathbb{Z}^{r}$,

$$
\psi(\underline{\underline{u}})=h\left(\alpha_{1}^{u_{1}} \cdots \alpha_{r}^{u_{r}}\right)+h\left(\beta_{1}^{u_{1}} \cdots \beta_{r}^{u_{r}}\right)=h(x)+h(y)=h_{s}(\underline{\underline{x}})
$$

where $\underline{\underline{x}}$ is given by $(3.1)$. We have

(a) $\psi(\underline{\xi}) \geqq 0$,

(b) $\psi(\bar{\alpha} \underline{\xi})=|\alpha| \psi(\underline{\xi})$ for $\alpha \in \mathbb{R}$,

(c) $\psi(\underline{\underline{\xi}}+\underline{\underline{\eta}}) \leqq \psi(\underline{\underline{\bar{\xi}}})+\psi(\underline{\underline{\eta}})$.

We may infer that $\psi$ is continuous. The set $\Psi$ consisting of points $\underline{\underline{\xi}} \in \mathbb{R}^{r}$ with $\psi(\underline{\underline{\xi}}) \leqq 1$ is convex, symmetric (i.e., $\underline{\underline{\xi}} \in \Psi$ yields $-\underline{\underline{\xi}} \in \Psi$ ), closed, and contains $\underline{\underline{0}}$ in its interior.

Lemma 3. Suppose $\psi: \mathbb{R}^{r} \rightarrow \mathbb{R}$ with (a), (b), (c) has

$$
\psi(\underline{\underline{u}}) \geqq c>0
$$

for every $\underline{\underline{u}} \in \mathbb{Z}^{r} \backslash\{\underline{\underline{0}}\}$, and a fixed constant $c$. Then the set $\Psi$ is compact.

Proof. Pick $\alpha$ in $0<\alpha<c$. Then $\alpha \Psi$ contains no nonzero integer point, hence has finite volume by Minkowski's Theorem. Therefore $\Psi$ has finite volume, and since it is convex and contains $\underline{\underline{0}}$ in its interior, it is bounded, hence compact.

Lemma 4. Suppose $\psi$ satisfies (3.2). Let $\mathfrak{U} \subseteq \mathbb{R}^{r}$ be a set of points such that

$$
\psi(\underline{\underline{u}}-\underline{\underline{v}}) \geqq \delta_{0}>0
$$

for $\underline{\underline{u}} \neq \underline{\underline{v}}$ in $\mathfrak{U}$. Then the number of $\underline{\underline{u}} \in \mathfrak{U}$ with

$$
\psi(\underline{\underline{u}}) \leqq C
$$

is

$$
\leqq\left(\left(2 C / \delta_{0}\right)+1\right)^{r}
$$


Proof. Let $\Psi(\underline{\underline{u}})$ be the set $\frac{1}{2} \delta_{0} \Psi+\underline{\underline{u}}$, i.e., the set $\frac{1}{2} \delta_{0} \Psi$ translated by $\underline{\underline{u}}$. By our hypothesis, sets $\Psi(\underline{\underline{u}}), \Psi(\underline{\underline{v}})$ with $\underline{\underline{u}} \neq \underline{\underline{v}}$ in $\mathfrak{U}$ have no interior points in common. On the other hand, when $\psi(\underline{\underline{u}}) \leqq C$, the set $\Psi(\underline{\underline{u}}) \subseteq\left(C+\frac{1}{2} \delta_{0}\right) \Psi$. Comparing volumes (which are finite by the preceding lemma) we see that the number of points $\underline{\underline{u}} \in \mathfrak{U}$ with $\psi(\underline{\underline{u}}) \leqq C$ is

$$
\leqq\left(C+\frac{1}{2} \delta_{0}\right)^{r} /\left(\frac{1}{2} \delta_{0}\right)^{r}=\left(\left(2 C / \delta_{0}\right)+1\right)^{r}
$$

The proof of Theorem 3 is now rapidly finished as follows. Let $\mathfrak{S}$ be the set of points whose cardinality is to be estimated. Call $\underline{\underline{x}}, \underline{\underline{x}}^{\prime}$ in $\mathfrak{S}$ neighbors if $\underline{\underline{x}} \neq \underline{\underline{x}}^{\prime}$ and

$$
\delta(\underline{\underline{x}}, \underline{\underline{x}})<c_{4}(P) / q .
$$

In view of Theorem 2 , an element $\underline{\underline{x}} \in \mathfrak{S}$ has $\leqq q-1$ neighbors. Pick $\underline{\underline{x}}_{1} \in \mathfrak{S}$, and let $\mathfrak{S}_{1}$ be obtained from $\mathfrak{S}$ by removing $\underline{\underline{x}}_{1}$ and its neighbors. If $\mathfrak{S}_{1}$ is nonempty, pick $\underline{\underline{x}}_{2} \in \mathfrak{S}_{1}$ and let $\mathfrak{S}_{2}$ be obtained from $\mathfrak{S}_{1}$ by removing $\underline{\underline{x}}_{2}$ and its neighbors, etc. This process will come to an end, and we obtain a "thinned out" set $\mathfrak{S}^{\prime}=$ $\left\{\underline{\underline{x}}_{1}, \ldots, \underline{\underline{x}}_{\ell}\right\} \subseteq \mathfrak{S}$ no two of whose elements are neighbors, with card $\mathfrak{S}^{\prime} \geqq q^{-1}$ card $\mathfrak{S}$. To $\mathfrak{S}^{\prime}$ corresponds a set $\mathfrak{U}$ of points $\underline{\underline{u}} \in \mathbb{Z}^{r}$ having (3.3) with $\delta_{0}=c_{4}(P) / q$. By Lemma 4,

$$
\operatorname{card} \mathfrak{U} \leqq\left(\left(2 C q / c_{4}(P)\right)+1\right)^{r} \leqq\left(3 C q / c_{4}(P)\right)^{r}
$$

when $C \geqq 1$. Then

$$
\operatorname{card} \mathfrak{S} \leqq q \text { card } \mathfrak{S}^{\prime}=q \operatorname{card} \mathfrak{U} \leqq q\left(3 C q / c_{4}(P)\right)^{r} .
$$

Therefore when $\Gamma$ is finitely generated, Theorem 3 holds with

$$
c_{5}(P)=3 q / c_{4}(P) \leqq 3 p \tilde{p} \cdot 2^{8 p m-4} \leqq 6 p^{2} \cdot 2^{8 p m-4}<2^{9 p m},
$$

since (as is clear from the proof of Theorem 2) we may take $c_{4}(P)=2^{4-8 p m}$, and since $\tilde{p} \leqq 2 p$.

In general, $\Gamma$ is a union of finitely generated groups $\Gamma_{1} \subseteq \Gamma_{2} \subseteq \cdots$ of rank $r$. Our estimate holds for each $\Gamma_{i}(i=1,2, \ldots)$, hence also for $\Gamma$.

Theorem 3a. When $P_{0}=u X+v Y+w$ with nonzero coefficients, the number of zeros $\underline{\underline{x}}$ of $P_{0}$ with $\underline{\underline{x}} \in \Gamma$ and $h_{s}(\underline{\underline{x}}) \leqq C$, where $C \geqq 1$, is

$$
\leqq 2 \cdot(210 C)^{r} \text {. }
$$

Proof. Here $p=1, \tilde{p}=2$, and we may take $c_{4}(P)=1 / 52$. Therefore (3.4) becomes

$$
\operatorname{card} \mathfrak{U} \leqq(208 C+1)^{r}<(210 C)^{r},
$$

and

$$
\operatorname{card} \mathfrak{S} \leqq 2 \operatorname{card} \mathfrak{S}^{\prime} \leqq 2(210 C)^{r}
$$




\section{A fuRther RESUlt}

For applications in subsequent work [4], it will be convenient to give the following easy

Theorem 4. Let $K$ be a number field of degree $d$, and $\Gamma \subseteq\left(K^{\times}\right)^{n}$ of rank $r$. Given $C \geqq 1$, the number of $\underline{\underline{x}} \in \Gamma$ with $h_{s}(\underline{\underline{x}}) \leqq C$ is

$$
\leqq\left(2 d^{2}\right)^{n}\left(43 d^{3} C\right)^{r}
$$

Proof. Call $\underline{\underline{x}} \neq \underline{\underline{x}}$ in $\Gamma$ neighbors if $\underline{\underline{x}} / \underline{\underline{x}} \in U^{n}$. Now if the roots of 1 in $K$ form a group of order $k$, we have $\Phi(k) \leqq \bar{d}$. Since $\Phi(k) \geqq(k / 2)^{1 / 2}$, we have $k \leqq 2 d^{2}$. Therefore $\underline{\underline{x}}$ has $<k^{n} \leqq\left(2 d^{2}\right)^{n}$ neighbors. When $\underline{\underline{x}} \neq \underline{\underline{x}}^{\prime}$ are not neighbors, we have for $d>1$,

$$
\delta\left(\underline{\underline{x}}, \underline{\underline{x}}^{\prime}\right)=h\left(\underline{\underline{x}} / \underline{\underline{x}}^{\prime}\right)>\log \left(1+\frac{\log d}{6 d^{2}}\right)^{1 / d}>\log \left(1+\frac{1}{20 d^{3}}\right)>\frac{1}{21 d^{3}}
$$

by a very generous minorization of an estimate of Dobrowolski [1]. Therefore setting $\delta_{0}=1 / 21 d^{3}$ and proceeding as for Theorem 3 , we obtain

$$
\text { card } \mathfrak{S} \leqq\left(2 d^{2}\right)^{n}\left(\left(2 C / \delta_{0}\right)+1\right)^{r}=\left(2 d^{2}\right)^{n}\left(42 d^{3} C+1\right)^{r} .
$$

\section{REFERENCES}

1. E. Dobrowolski, On a question of Lehmer and the number of irreducible factors of a polynomial, Acta Arith. 34 (1979), 391-401. MR 80i:10040

2. D. H. Lehmer, Factorization of certain cyclotomic functions, Ann. Math. 34 (2) (1933), 461479 .

3. H. P. Schlickewei, Equations ax $+b y=1$, Annals of Math., (to appear).

4. H. P. Schlickewei and W. M. Schmidt, Linear equations in variables which lie in a multiplicative group, In preparation.

5. H. P. Schlickewei and E. Wirsing, Lower bounds for the heights of solutions of linear equations, Invent. Math, (to appear).

6. W. M. Schmidt, Diophantine Approximation, Springer Lecture Notes in Mathematics $\mathbf{7 8 5}$ (1980). MR 81j:10038

7. D. Zagier, Algebraic numbers close to both 0 and 1, Math. Computation 61 (1993), 485-491. MR 94c: 11104

8. S. Zhang, Positive line bundles on arithmetic surfaces, Ann. of Math. 136 (1992), 569-587. MR 93j:14024

Department of Mathematics, University of Colorado, Boulder, Colorado 80309-0395

E-mail address: Schmidt@Euclid.colorado.edu 